\title{
Primeiro registro de Aleurocanthus woglumi (Hemiptera: Aleyrodidae) no estado de Santa Catarina, Brasil
}

\author{
Rodolfo Vargas Castilhos ${ }^{1}$, Eduardo Cesar Brugnara ${ }^{1}$, Rafael Roveri Sabião ${ }^{1}$, \\ Thomaz Palma Rocha Andrade ${ }^{2} \&$ Gilmar Negri ${ }^{3}$
}

\begin{abstract}
RESUMO
O objetivo deste trabalho foi relatar pela primeira vez a ocorrência da mosca-negra-dos-citros Aleurocanthus woglumi (Hemiptera: Aleyrodidae) no estado de Santa Catarina, Brasil. Folhas e brotos de laranjeira Caipira e Valência e tangerineira Ponkan foram coletados no município de Coronel Freitas, na região oeste do estado, em abril de 2019 e analisados em laboratório. Foram observados ovos, ninfas e adultos da praga. Em Santa Catarina existem mais de 1.900 ha de pomares cítricos comerciais, com grande relevância para a região oeste, onde as plantas cítricas também são frequentes em pomares domésticos. Assim, ações de monitoramento e manejo integrado são necessárias para minimizar os potenciais danos de A. woglumi à citricultura catarinense.
\end{abstract}

Termos de indexação: praga sugadora, mosca-negra-dos-citros, citricultura, distribuição geográfica.

\section{First record of Aleurocanthus woglumi (Hemiptera: Aleyrodidae) in the state of Santa Catarina, Brazil}

\section{SUMMARY}

The objective of this work was to report for the first time the occurrence of the citrus blackfly Aleurocanthus woglumi (Hemiptera: Aleyrodidae) in the state of Santa Catarina, Brazil. Leaves and shoots of Caipira and Valência sweet oranges and Ponkan tangerine were collected in the municipality of Coronel Freitas, located in the western region of the state, in April 2019, and analyzed in laboratory. Eggs, nymphs and adults of the pest were observed. In Santa Catarina there are more than 1,900 ha of commercial citrus orchards, with great relevance in the western region, where citrus plants are also frequent in domestic orchards. Monitoring and integrated management actions are required to minimize the potential damage that $A$. woglumi can cause to Santa Catarina citriculture.

Index terms: sucking pest, citrus blackfly, citriculture, geographical distribution.

\footnotetext{
${ }^{1}$ Centro de Pesquisa para Agricultura Familiar, Empresa de Pesquisa Agropecuária e Extensão Rural de Santa Catarina - EPAGRI, Chapecó, SC, Brasil

${ }^{2}$ Goldcitrus Consultoria e Representações, Chapecó, SC, Brasil

${ }^{3}$ Alamos Brasil Agroquímicos, Pinhalzinho, SC, Brasil

Autor correspondente: Rodolfo Vargas Castilhos, Empresa de Pesquisa Agropecuária e Extensão Rural de Santa Catarina - EPAGRI, Servidão Ferdinando Ricieiri Tusset, s/n, CEP 89803-904, Chapecó, SC, Brasil. E-mail: rodolfocastilhos@epagri.sc.gov.br
} 
Aleurocanthus woglumi Ashby, 1915 (Hemiptera: Aleyrodidae), conhecida como mosca-negra-dos-citros (MNC), é uma importante praga da citricultura mundial, com ocorrência em regiões tropicais e subtropicais do globo, tais como África, Índia Ocidental, Oceania e Américas Central, do Norte e do Sul (Silva et al., 2011). No continente sul-americano, a MNC já foi encontrada na Venezuela, Colômbia, Equador, Suriname, Peru, Guiana, Paraguai, Argentina e Brasil (López et al., 2011; Silva et al., 2011; Vieira et al., 2017).

Este inseto teve sua ocorrência relatada pela primeira vez no Brasil em 2001, no estado do Pará, e atualmente se encontra disseminado por quase todo o País (Raga et al., 2013; Maciel, 2015). A MNC está presente, seja em pequenos focos ou em áreas de produção comercial de citros, com relatos de ocorrência em praticamente todos os estados da Federação brasileira, com exceção do Acre, de Santa Catarina, do Rio Grande do Sul e do Distrito Federal (Maciel, 2015; Vieira et al., 2017). Por mais de uma década, A. woglumi foi considerada praga quarentenária presente no Brasil, porém com a expansão de sua ocorrência no território nacional, foi excluída da lista de pragas quarentenárias A2 por meio da Instrução Normativa ${ }^{\circ} 42$, de 9 de dezembro de 2014 (Brasil, 2014), visto estar presente em 23 dos 27 estados brasileiros.

Este trabalho objetivou relatar pela primeira vez a ocorrência de A. woglumi (MNC) no estado de Santa Catarina.

No dia 04 de abril de 2019, em visita a uma propriedade produtora de citros localizada no município de Coronel Freitas, SC (26⒌'53,4”S, 5243'16,5”O), brotações infestadas com ovos, ninfas e adultos de inseto suspeito de ser a MNC foram coletadas, em diferentes pontos do pomar. A coleta se deu em plantas de laranjeira doce Caipira [Citrus sinensis (L.) Osbeck] (pé franco), com aproximadamente 10 anos de idade (à altitude de 558m), de tangerineiras Ponkan e Montenegrina (Citrus reticulata Blanco), sobre porta enxerto Poncirus trifoliata (L.) Raf., com aproximadamente 5 anos de idade (à altitude de $558 \mathrm{~m}$ ), e laranjeira doce 'Folha Murcha' (C. sinensis), sobre porta enxerto citrumeleiro Swingle (Citrus paradisi Macf. $\times$ P. trifoliata), com aproximadamente 5 anos de idade (à altitude de $603 \mathrm{~m}$ ).

O material coletado foi enviado ao Laboratório de Fitossanidade da Empresa de Pesquisa Agropecuária e Extensão Rural de Santa Catarina (Epagri/Cepaf), em Chapecó-SC, onde foi analisado e o inseto identificado. A espécie foi identificada se tratar da $\mathrm{MNC}$ (A. woglumi) com base em caracteres morfológicos, conforme descrito por Nguyen et al. (2019). Após registro fotográfico, espécimes voucher foram depositados na coleção entomológica da Epagri/Cepaf.

Os adultos da MNC apresentavam corpo avermelhado, com asas de coloração cinza-escura, com aproximadamente $1 \mathrm{~mm}$ de comprimento (Figura 1A). Os ovos possuíam coloração amarelada-alaranjada e foram depositados em forma de espiral na face abaxial da folha (Figura 1B). As ninfas se caracterizavam pela coloração negra e numerosos "espinhos", que surgem a partir do segundo instar (Figura 1C), concordando com a descrição de Raga et al. (2013). De acordo com Silva et al. (2011), o ciclo ovo-adulto pode levar de 43 a 126 dias, dependendo da temperatura.

Adultos da MNC podem infestar mais de 300 espécies de plantas, como mangueira, cajueiro, abacateiro, mandioca, goiabeira, pitangueira, aceroleira, plantas ornamentais, entre outras (Raga et al., 2013; Maciel, 2015; Nguyen et al.,

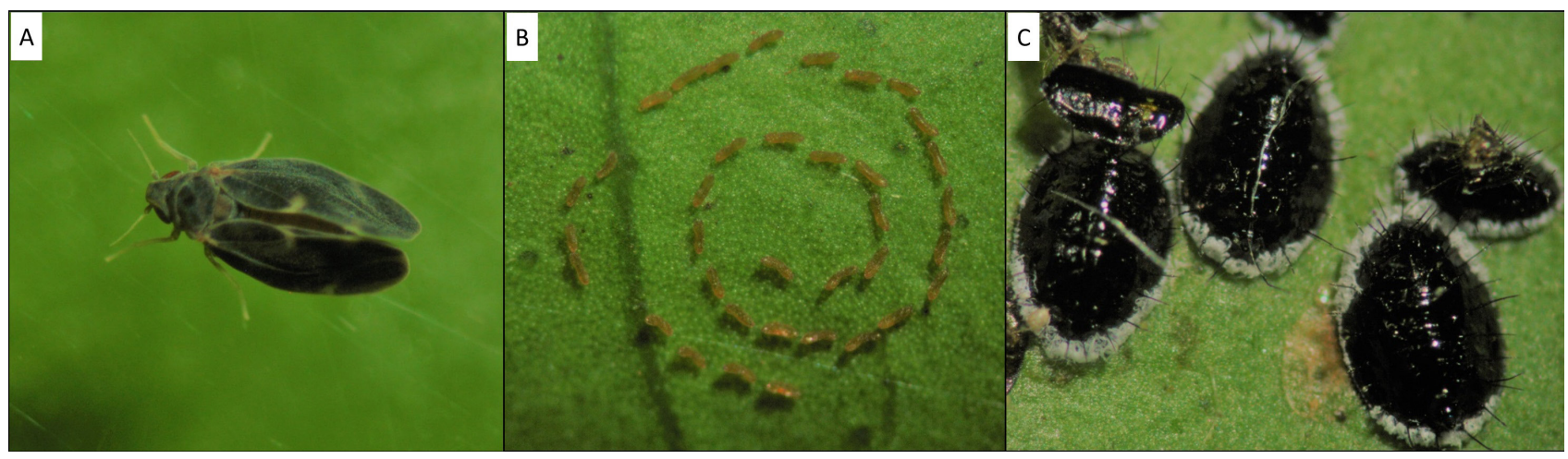

Figura 1. Adulto (A), postura em espiral (B) e ninfas de $4^{\circ}$ instar (C) de Aleurocanthus woglumi. Fotos: Rodolfo Vargas Castilhos. 
2019). Assim, o transporte do inseto em mudas não cítricas é uma hipótese plausível para explicar a sua introdução em Santa Catarina.

Os danos diretos nos citros se dão pela sucção de seiva da planta, que leva ao seu enfraquecimento. Indiretamente, há a formação de fumagina sobre ramos, folhas e frutos infestados (Figura 2), o que causa redução da respiração e fotossíntese (Nguyen et al., 2019). Altas infestações da MNC podem resultar em perdas de produção, redução da qualidade dos frutos e aumento de custos de produção em função de eventuais pulverizações (Maciel, 2015). De acordo com Silva et al. (2011), as perdas na produção ocasionadas pela MNC podem variar de $20 \%$ a $80 \%$. Entre as espécies cítricas, há diferenças de preferência de espécies pela MNC para oviposição, sendo a laranjeira doce Rubi preferida em relação à tangerineira Ponkan e a limeira-ácida Tahiti é intermediária (Lopes et al., 2013).

Em termos globais, o estado de Santa Catarina tem 1.907 hectares de citros, cujo valor bruto da produção alcançou R\$16,7 milhões em 2017 (Empresa de Pesquisa Agropecuária e Extensão Rural de Santa Catarina, 2019). Na Figura 3 é apresentada a distribuição da produção de citros por município no Estado.

O município de Coronel Freitas, onde se registra pela primeira vez a ocorrência da MNC, localiza-se na
Mesorregião Oeste Catarinense, no vale do Rio Chapecó (Figura 3). Nesta região predomina o clima subtropical (Cfa, de Köppen), com ótima aptidão para cultivo de citros devido às geadas fracas e pouco frequentes, e à alternância de temperaturas diurnas (altas) e noturnas (amenas), que conferem ótimo aspecto e sabor aos frutos cítricos (Koller \& Soprano, 2013). Coronel Freitas tem 30 hectares cultivados com laranjeiras e 20 ha de tangerineiras. Incluindo os municípios vizinhos da microrregião de Chapecó, que vai desde o Rio Uruguai até a divisa com o estado do Paraná, são cultivados 285,7; 41,7 e 2,5 hectares de laranjeiras, tangerineiras e limoeiros, respectivamente. Outros 576 hectares são cultivados nas microrregiões de São Miguel do Oeste, Xanxerê e Concórdia, adjacentes à de Chapecó (Empresa de Pesquisa Agropecuária e Extensão Rural de Santa Catarina, 2019).

Outro fator que reforça a importância da ocorrência da MNC em Santa Catarina é a presença de plantas cítricas nos pomares domésticos da mesorregião oeste. Mais de 70\% das propriedades rurais têm plantas cítricas cultivadas para consumo da família e venda ocasional de excedentes (Nesi et al., 2019). Os pomares domésticos raramente são pulverizados com produtos fitossanitários para o controle de pragas, bem como muitos pomares comerciais, pois, entre outros motivos, não há registro de

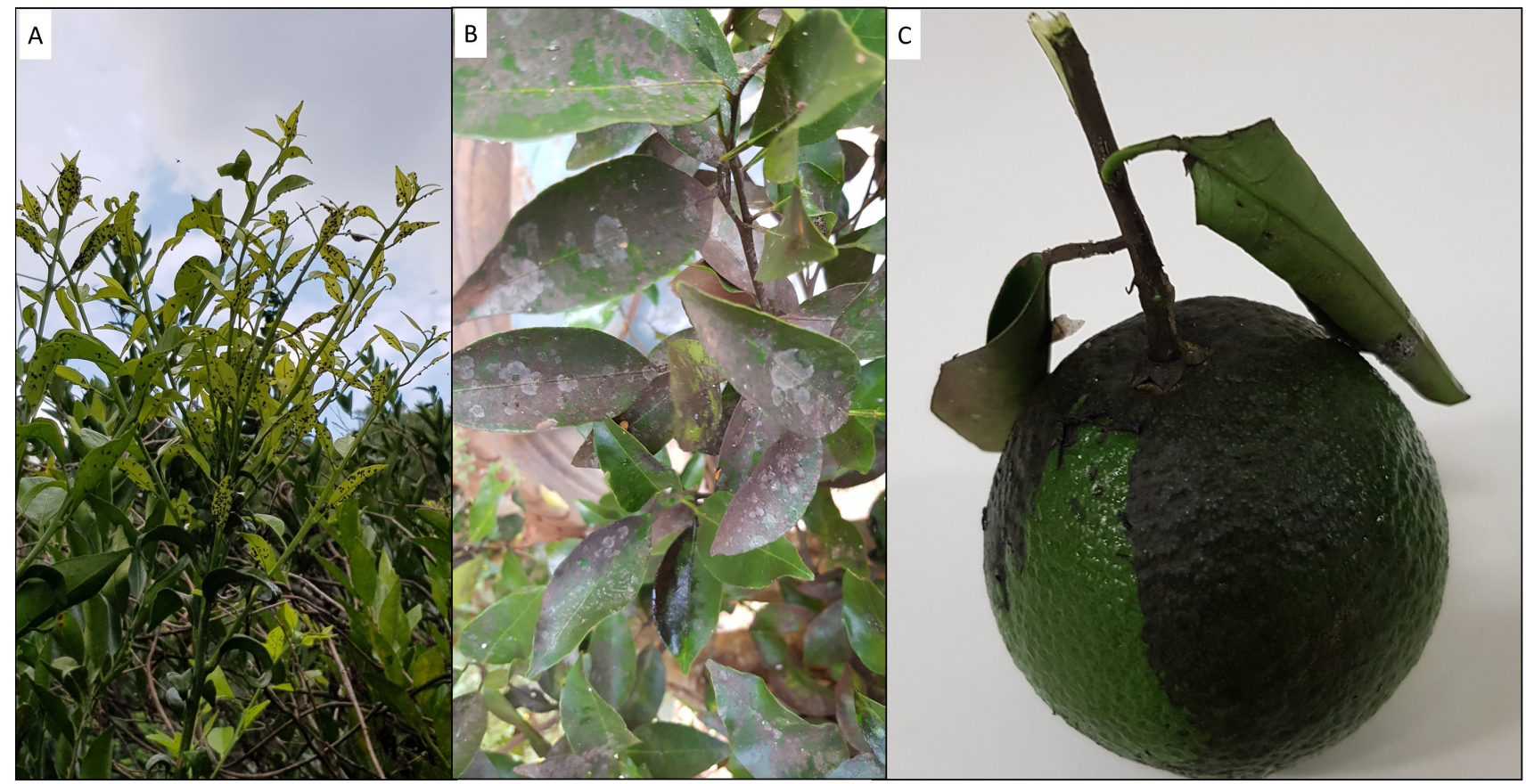

Figura 2. Danos de Aleurocanthus woglumi em citros: brotações infestadas com adultos (A) e fumagina em folhas (B) e fruto (C). Coronel Freitas-SC, 2019. Fotos: Rodolfo Vargas Castilhos. 


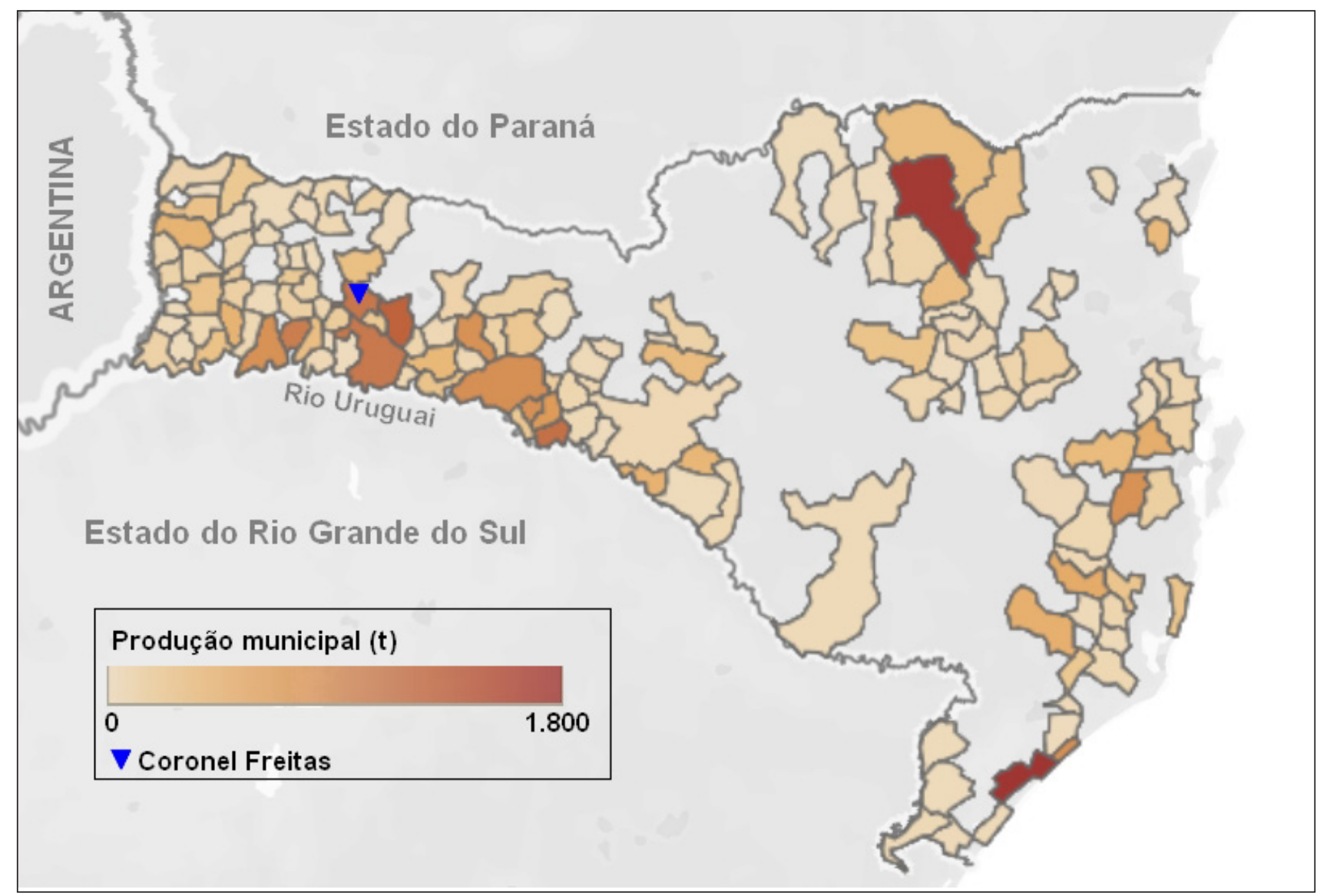

Figura 3. Distribuição da produção de frutas cítricas (laranjas, limões, limas e tangerinas) por município do estado de Santa Catarina. Adaptado de Epagri/Cepa (Empresa de Pesquisa Agropecuária e Extensão Rural de Santa Catarina, 2019).

ocorrência da bactéria Candidatus Liberibacter asiaticus, agente causal do huanglongbing (HLB) dos citros, que em regiões ocorrentes demanda intensas pulverizações de inseticidas para controle do inseto vetor (Canale et al., 2019; Dorta et al., 2019).

Além de ser um problema para a citricultura catarinense, a ocorrência da MNC no Oeste de Santa Catarina configura um risco para a dispersão dela para o Rio Grande do Sul, já que entre Coronel Freitas e a divisa com esse estado há pomares comerciais e domésticos de citros. A área colhida de laranjeiras, limoeiros e tangerineiras no Rio Grande do Sul em 2017 foi de cerca de 35 mil hectares, cujo valor bruto da produção foi de aproximadamente 390 milhões de reais. São 12.726 hectares cultivados na região noroeste do Rio Grande do Sul (Instituto Brasileiro de Geografia e Estatística, 2019), que é separada da região oeste de Santa Catarina pelo Rio Uruguai.

Diante do potencial risco que $A$. woglumi oferece à citricultura do estado de Santa Catarina, esforços futuros devem ser alocados no monitoramento da sua ocorrência nas principais regiões citrícolas, quantificação dos danos e estabelecimento de um plano de manejo integrado regional, a fim de evitar o aumento da sua disseminação e minimizar os impactos negativos na produção citrícola catarinense.

\section{AGRADECIMENTOS}

À Zelinda Meneguzzi, técnica de laboratório da Epagri/Cepaf, pelo auxílio na captura de imagens da MNC.

\section{REFERÊNCIAS}

Brasil. Ministério da Agricultura, Pecuária e Abastecimento. (2014, 10 de dezembro). Instrução Normativa no 42, de 9 de dezembro de 2014. Exclui da Lista de Pragas Quarentenárias Presentes - (A2) o inseto Aleurocanthus 
woglumi (Mosca Negra dos Citros), constante do Anexo II da Instrução Normativa no 41 e alterado pela Instrução Normativa. Brasília, DF: Diário Oficial da República Federativa do Brasil, no. 59, seção 1.

Canale, M. C., Castilhos, R. V., Maro, L. A. C., \& Brugnara, E. C. (2019). Monitoramento de Diaphorina citri, vetor da bactéria causadora do Huanglongbing dos citros, em Santa Catarina. In Anais do Simpósio de Fruticultura da Região Sul - FRUSUL (Vol. 2, No. 1). Chapecó: UFFS. Recuperado em 9 de julho de 2019, de https://portaleventos.uffs.edu.br/index.php/FRUSUL/ article/view/10735

Dorta, S. O., Machado, M. A., \& Freitas-Astúa, F. (2019). Desenvolvimento de estratégias alternativas visando ao controle do huanglongbing. Citrus Research \& Technology, 40, e-1045.

Empresa de Pesquisa Agropecuária e Extensão Rural de Santa Catarina - Epagri. Centro de Socioeconomia e Planejamento Agrícola - CEPA. (2019). Painéis de Dados da Fruticultura. Levantamento de dados sobre a Fruticultura Catarinense - 2016/17. Florianópolis: Epagri/ Cepa. Recuperado em 14 de junho de 2019, de <https:// cepa.epagri.sc.gov.br/index.php/publicacoes/fruticultura/ paineis-fruticultura/

Instituto Brasileiro de Geografia e Estatística - IBGE. (2019). Sistema IBGE de Recuperação Automática SIDRA. Fornece dados estatísticos da Produção Agrícola Municipal do Brasil. 2018. Recuperado em 14 de junho 2019, de https://sidra.ibge.gov.br/tabela/1613

Koller, O. L., \& Soprano, E. (2013). Planejamento do pomar. In O. L. Koller (Org.), Citricultura catarinense (pp. 41-56). Florianópolis: Epagri.

Lopes, G. S., Lemos, R. N. S., Araujo, J. R. G., Marques, L. J. P., \& Vieira, D. L. (2013). Preferência para oviposição e ciclo de vida de mosca-negra- dos-citros Aleurocanthus woglumi Ashby em espécies frutíferas. Revista Brasileira de Fruticultura, 35(3), 738-745.
López, S. N., Peralta, C., Aguirre, A., \& Cáceres, S. (2011). Primer registro de "la mosca negra de los cítricos" Aleurocanthus woglumi (Hemiptera: Aleyrodidae) en la Argentina. Revista de la Sociedad Entomológica Argentina, 70(3-4), 373-374.

Maciel, I. L. (2015). Status sanitário da mosca negra dos citros no Brasil (Dissertação de mestrado). Universidade Federal de Viçosa, Viçosa.

Nesi, C. N., Dorigon, C., \& Pierezan, S. (2019). Produção de frutas para autoconsumo na região Oeste Catarinense. In Anais do Simpósio de Fruticultura da Região Sul FRUSUL (Vol. 2, No. 1). Chapecó: UFFS. Recuperado em 2 de julho de 2019, de https://portaleventos.uffs.edu. br/index.php/FRUSUL/article/view/10692

Nguyen, R., Hamon, A. B., \& Fasulo, T. (2019). Citrus blackfly, Aleurocanthus woglumi Ashby (Insecta: Hemiptera: Aleyrodidae) (4 p.). Gainesville: University of Florida, IFAS Extension.

Raga, A., Imperato, R., Mello, W. J., \& Maia, S. (2013). Mosca negra dos citros. Citrus Research \& Technology, 34(2), 57-63.

Silva, A. G., Farias, P. R. S., Boiça Junior, A. L., \& Souza, B. H. S. (2011). Mosca-negra-dos-citros: características gerais, bioecologia e métodos de controle dessa importante praga quarentenária da citricultura brasileira. EntomoBrasilis, 4(3), 85-91.

Vieira, D. L., Batista, J. L., Oliveira, R., Malaquias, J. B., \& Souza, G. M. M. (2017). Aleurocanthus woglumi (Hemiptera: Aleyrodidae) in Citrus: opportunities and challenges to implement a sustainable management (135 p.). London: IntechOpen.

Recebido: Julho 16, 2019

Aceito: Outubro 22, 2019

Como citar: Castilhos, R. V., Brugnara, E. C., Sabião, R. R., Andrade, T. P. R., $\&$ Negri, G. (2019). Primeiro registro de Aleurocanthus woglumi (Hemiptera: Aleyrodidae) no estado de Santa Catarina, Brasil. Citrus Research \& Technology, 40, e1051. https://doi.org/10.4322/crt.18919 